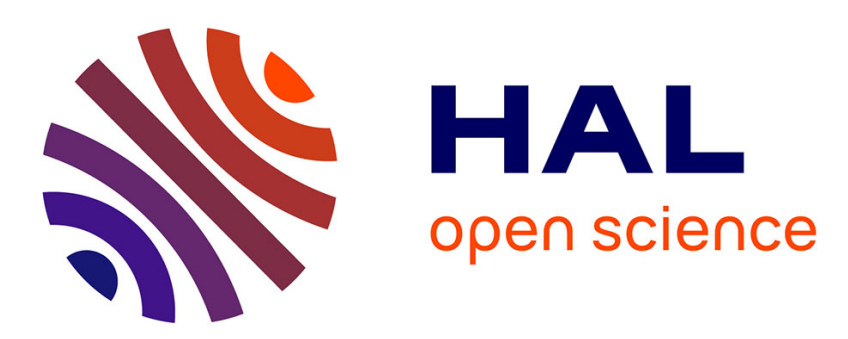

\title{
The SG2 algorithm for a fast and accurate computation of the position of the Sun for multi-decadal time period Philippe Blanc, Lucien Wald
}

\section{To cite this version:}

Philippe Blanc, Lucien Wald. The SG2 algorithm for a fast and accurate computation of the position of the Sun for multi-decadal time period. Solar Energy, 2012, 88 (10), pp.Pages 3072-3083. 10.1016/j.solener.2012.07.018 . hal-00725987

\section{HAL Id: hal-00725987}

https://hal-mines-paristech.archives-ouvertes.fr/hal-00725987

Submitted on 16 Oct 2012

HAL is a multi-disciplinary open access archive for the deposit and dissemination of scientific research documents, whether they are published or not. The documents may come from teaching and research institutions in France or abroad, or from public or private research centers.
L'archive ouverte pluridisciplinaire HAL, est destinée au dépôt et à la diffusion de documents scientifiques de niveau recherche, publiés ou non, émanant des établissements d'enseignement et de recherche français ou étrangers, des laboratoires publics ou privés. 


\title{
THE SG2 ALGORITHM FOR A FAST AND ACCURATE COMPUTATION OF THE POSITION OF THE SUN FOR MULTI- DECADAL TIME PERIOD
}

Ph. Blanc, L. Wald

Centre Energétique et Procédés, MINES ParisTech, BP 207, 06904, Sophia Antipolis cedex, France

Corresponding author: Philippe Blanc, philippe.blanc@mines-paristech.fr

\begin{abstract}
The solar position algorithm (SPA) is a very accurate but slow algorithm for the computation of the Sun position with respect to an observer at ground surface. We compare the results of three fast algorithms to the SPA and establish their performances. We propose a new algorithm SG2 that is faster than the three others and offers the same level of accuracy than the most accurate, i.e., maximum error in solar vector of order of 10", for a multi-decadal time period, with an example of a 50-year period: 1980-2030. This performance is achieved by devising approximations of the original equations of the SPA to decrease the number of operations. This yields a decrease in accuracy that is controlled and bounded. The mathematical tools permitting to determine these approximations with a selected uncertainty level are described.
\end{abstract}

Keywords: Sun position; Solar azimuth angle; Solar zenithal angle; Declination; Universal Time; Solar radiation.

\section{NOMENCLATURE AND ABREVIATIONS}

Time references

TT Terrestrial Time (s)

TAI International Atomic Time (TAI: Temps Atomique International) (s)

UT Universal time (s). Also called UT1

UTC Coordinated universal time (s)

$\Delta_{\mathrm{TT}} \quad$ Difference between TT and UT (s)

$j_{T T} \quad$ Terrestrial julian date (decimal day, d)

$j_{U T} \quad$ Universal julian date (d) 
$j_{T T}^{*} \quad$ Modified terrestrial julian date, defined from 1980-01-01T00:00:00 TT(d)

$j_{U T}^{*} \quad$ Modified universal julian date, defined from 1980-01-01T00:00:00 UT (d)

\section{Solar radiation}

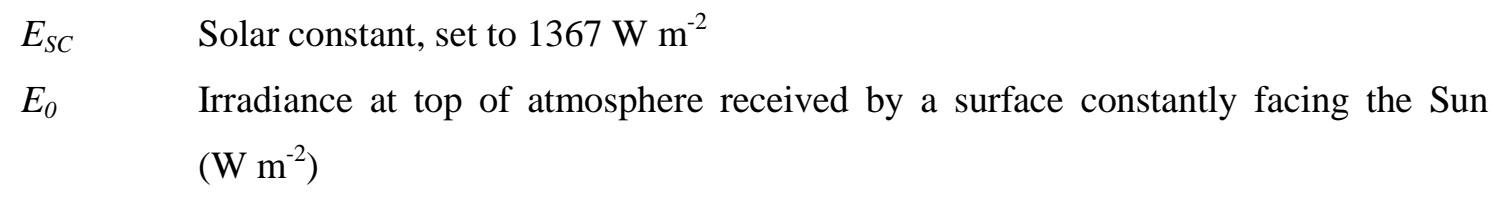

\section{Heliocentric coordinates of the Earth}

AU Astronomical unit is a unit of length equal to $149597870691 \mathrm{~m} \pm 6 \mathrm{~m}$ (McCarthy et Petit, 2003), corresponding to the yearly mean of the Earth-Sun distance.

$R \quad$ Earth heliocentric radius (AU)

$L \quad$ Earth heliocentric longitude (rad)

$B \quad$ Earth heliocentric latitude (rad)

Geocentric coordinates of the Sun

$\Theta_{a} \quad$ Apparent Sun geocentric longitude (rad)

$\Delta \tau \quad$ Stellar aberration correction (rad)

$\Delta \psi \quad$ Nutation in Sun geocentric longitude (rad)

$\varepsilon \quad$ True Earth obliquity (rad)

$r_{\alpha}^{g} \quad$ Sun geocentric right ascension (rad)

$\delta^{g} \quad$ Sun geocentric declination (rad)

$\omega^{5} \quad$ Geocentric hour angle (rad)

Quantities related to a local observer on Earth

$\varphi \quad$ Geographical latitude of the observer (rad). It refers to a reference geographical ellipsoid of the Earth

$\lambda \quad$ Geographical longitude of the observer (rad). It refers to a reference geographical ellipsoid of the Earth

$h \quad$ Altitude or elevation of the local observer above the reference geographical ellipsoid (m)

a Semi-major axis of the reference geographical ellipsoid, or equatorial axis (m)

f Earth flattening of the reference geographical ellipsoid (unitless)

$\mathrm{T} \quad$ Annual average of local air temperature $\left({ }^{\circ} \mathrm{C}\right)$

$\mathrm{P} \quad$ Annual average of local air pressure $(\mathrm{hPa})$

$\xi \quad$ Equatorial horizontal parallax of the Sun (rad) 


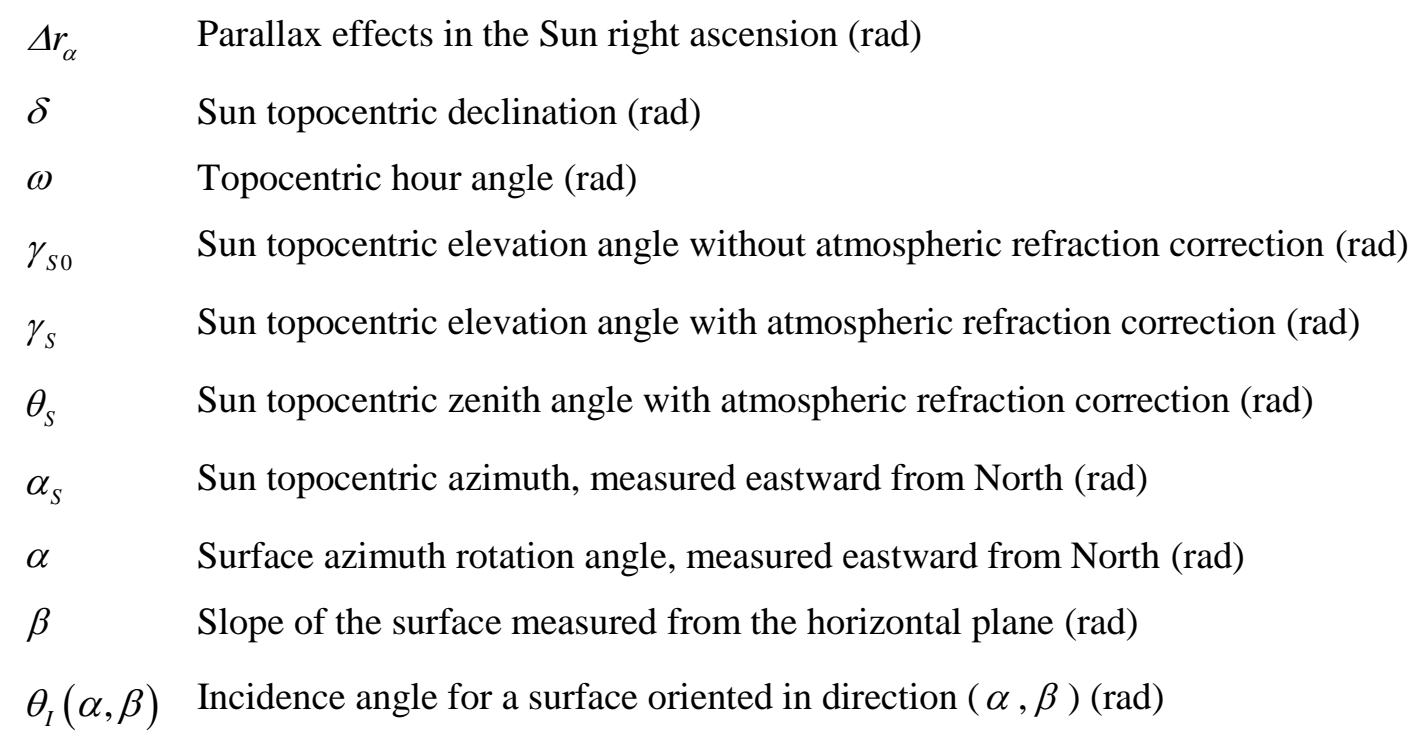

\section{Algorithms for Sun position}

SPA Sun Position Algorithm from the National Renewable Energy Laboratory (NREL) (Reda, Andreas 2003, 2004)

SG Algorithm Solar Geometry from the European Solar Radiation Atlas (ESRA, 2000; Wald, 2007)

ENEA Algorithm from Italian National Agency for New Technologies, Energy and Sustainable Economic Development (ENEA) (Grena, 2008)

MICH Algorithm from (Michalsky, 1988)

Statistical quantities for comparison

MBE Mean Bias Error

RMSE Root Mean Square Error

MAXE Maximum of Absolute Error

\section{INTRODUCTION}

At any time, the Earth intercepts approximately $180 \times 10^{6} \mathrm{GW}$ of the power emitted by the Sun. The amount of power received at a given geographical site at the Earth surface varies in time: between daynight due to the Earth's rotation and between seasons because of the Earth's orbit and Earth's axis inclination. At a given time it also varies in space, because of the changes in the obliquity of the solar rays with longitude and latitude. Accordingly, the amount of power received at a given location, plan orientation and time depends upon the relative position of the Sun and the Earth. This is why both Sun-Earth geometry and time play an important role in solar energy conversion and photo-energy systems. 
Technological advances and increasing deployment of such systems result in an increasing demand for more accurate knowledge on the position of the Sun with respect to a system. The demand may be direct, e.g., for pointing a concentrating device or a measuring instrument (Blanco-Muriel et al., 2001; Stafford et al., 2009), or indirect, e.g., for computing radiation quantities by models and exploitation of satellite images (Espinar et al., 2009; Perez et al., 2002; Rigollier et al., 2004).

The major rationale of our work on solar position takes place in the development of the HelioClim family of satellite-based surface solar irradiation databases, but without being limited to them. The HelioClim databases are initiatives of MINES ParisTech to increasing knowledge on downwards solar irradiance at surface (surface solar irradiance, SSI) for any site, any instant within a large geographical area and large period of time (Blanc et al., 2011; Cros et al., 2004, Diabate et al., 1989). The databases HelioClim cover Europe, Africa and the Atlantic Ocean. They are widely used by companies and practitioners (Gschwind et al., 2006). The database HelioClim-1 contains daily values of SSI for the period 1985-2005 and has been discussed in this journal by Lefèvre et al. (2007). It has been created from archives of images of the Meteosat First Generation, series of geostationary satellites. The database HelioClim-3 represents a step forward regarding spatial and time resolution, uncertainty on SSI and time to access to recent data (Blanc et al., 2011). It exploits the enhanced capabilities of the series of satellites Meteosat Second Generation to offer values of SSI every 15 min and every $3 \mathrm{~km}$.

Building upon the experience gained with the previous Meteosat (Diabaté et al., 1989), MINES ParisTech has a Meteosat Second Generation receiving station since 2003, and set up a routine operation for converting Meteosat data into global SSI on horizontal plane, in quasi-real time. Other parameters such as the different components of SSI received by a tilted plane are computed from the into global SSI on horizontal plane using, for example, the algorithms adopted by the European Solar Radiation Atlas (ESRA, 2000). These real-time operations creating 1-min averaged time series of SSI require computation of solar positions for each second within the minute. Accordingly, the requirements set on the algorithm for computing the solar position are:

- an accuracy better than $0.005^{\circ}\left(\sim 18^{\prime \prime}\right)$;

- a period of validity from 1980 to 2030 covering, with a margin, the planned periods of the HelioClim databases;

- a computational speed fast enough so that it can be used to process rapidly an image of 9 million pixels that requires approximately 1 millions of sun positions calculated in less than $1 \mathrm{~min}$.

These requirements for sun position calculation are not only for HelioClim. They may stand for other application as mentioned earlier for irradiation data processing such as quality control, global-to-direct conversion or solar-tracking devices. 
Accurate knowledge of Sun-Earth geometry and of time is one of the results reached in astronomy (Bretagnon, Francou, 1988; Meeus, 1999). Algorithms implementing this knowledge for solar radiation applications exist and have been published (Reda, Andreas, 2003, 2004). Their implementation is meant to calculate solar zenith and azimuth angles - and other related parameters in the period from -2000 to 6000 with standard deviation of $0.0003^{\circ}(1 ")$. In practice, time requested for computation may be excessive. Approximately 2300 floating operations and more than 300 direct and inverse trigonometric functions are used for one solar position calculation at a given location and time. In order to speed up computations, approximations of these equations are made, aiming at reducing the number of floating operations. Several articles have been published in the solar energy domain, documenting algorithms for computing the solar position with respect to an observer at a given location on the Earth (Blanco-Muriel et al., 2001; ESRA, 2000; Grena, 2008; Kambezidis, Tsangrassoulis, 1993; Michalsky, 1988; Pitman and Vant-Hull, 1978; Walraven, 1978). These algorithms differ in the attained accuracy, in their period of validity: from a few years to several thousands of years, and in the computation cost. Various strategies exist to reduce operations, such as reducing the period of validity still keeping a high accuracy (Blanco-Muriel et al., 2001; Grena, 2008; Kambezidis, Tsangrassoulis, 1993), or keeping a large period and reducing the accuracy (ESRA, 2000; Michalsky, 1988).

None of the proposed algorithms meets the requirements expressed above as discussed in next section. After having studied the possibilities of extending or enhancing the published algorithms, we have opted for a new algorithm whose description is one of the aims of this article. Another aim is to describe the mathematical tools permitting to determine the various approximations requested to achieve a given uncertainty level for a given time period. These tools should permit further development of other algorithms on Sun position meeting other requirements than those of HelioClim. Libraries in Matlab and C are proposed on the web to this purpose, and a Web service has been implemented.

\section{AVAILABLE ALGORITHMS AND THEIR PERFORMANCES}

Reda, Andreas $(2003,2004)$ propose the solar position algorithm, called SPA, for solar radiation applications. It is based on the VSOP87 solutions to planetary theories (Bretagnon and Francou, 1988) and the equations proposed by Meeus (1999). The uncertainty, in terms of standard deviation of solar azimuth and elevation angles is stated to be within $5 \mu \mathrm{rad}\left(0.0003^{\circ}\right.$, or $\left.1 "\right)$ for a very large period from -2000 to 6000. Libraries in C, Matlab and Python that implement the SPA algorithm are available (respectively at: rredc.nrel.gov/solar/codesandalgorithms/spa, www.mathworks.com/matlabcentral/fileexchange/4605 and www.pysolar.org). The number of operations required for one computation of the solar position is approximately 1000 additions, 1300 
multiplications and 300 calls of direct or inverse trigonometric functions. This algorithm is not fast enough for our purpose: an implementation in C of SPA on a 8-CPU machine would take approximately $2 \mathrm{~h}$ for the computation of the solar position every second within 15 min for the 9 millions of usable pixel of the HelioClim-3 database. Nevertheless, its accuracy is very high and we have selected the SPA as a reference against which three other algorithms are tested. The SPA constitutes the reference on which our approximations are constructed as well.

Currently, the HelioClim process exploits the solar position algorithm of the European Solar Radiation Atlas (ESRA, 2000; Wald, 2007). This algorithm, called hereinafter SG (Solar Geometry), is defined for the time period starting from 1980 and is considered as a fast solar position algorithm: it requires, for one solar position at one location and one time, approximately 25 additions, 35 multiplications and 25 calls of direct or inverse trigonometric functions. The time for the computation of the solar positions every second within 15 min for the 9 millions of usable pixels of the HelioClim-3 database is less than 8 minutes, compared to the $2 \mathrm{~h}$ needed for SPA.

We have tested two other fast algorithms. The algorithm of Michalsky (1988), called hereafter MICH, offers an uncertainty of approximately $0.01^{\circ}(\sim 40 ")$ in term of root mean square error, for the period 1950 to 2050. It requires approximately 20 additions, 35 multiplications and 25 calls of direct or inverse trigonometric functions. The fast algorithm proposed by Grena (2008), called hereafter ENEA, offers an uncertainty of $0.001^{\circ}(\sim 4 ")$, for the period 2003-2022. It requires approximately 40 additions, 40 multiplications and 25 calls of direct or inverse trigonometric functions. We have not retained that of Muriel-Blanco et al. (2001) because the uncertainty is close to that of MICH, but for a more restricted period: 1999 to 2015. In term of computation time, these two fast algorithms are more than 20 times faster than SPA.

To assess the performances in accuracy and precision of each algorithm in the time period 1980 to 2030, we have considered the SPA as a reference, and have computed the differences between the SPA and each of the three others, for 20000 dates randomly sampled between 1980 and 2030, for daylight time, at location $45^{\circ} \mathrm{N}$ and longitude $0^{\circ}$, at sea level. The solar position errors are computed for the solar azimuth $\Delta \alpha_{S}$ and the solar zenithal angle $\Delta \theta_{S}$, as well as for the solar vector $\Delta V_{S}$ defined by Grena (2008) as:

$$
\Delta V_{s}=\sqrt{\Delta \theta_{S}^{2}+\left(\Delta \alpha_{S} \sin \theta_{S}\right)^{2}}
$$

The errors are then summarized by a few statistical quantities: mean bias error (MBE), root mean square error (RMSE) and maximum of absolute error (MAXE), for each algorithm and each solar position errors (Table 1). 


\begin{tabular}{ccccc}
\hline \multirow{2}{*}{$\begin{array}{c}\text { Algorithm } \\
\text { SG } \\
1980-2030\end{array}$} & Errors & MBE & RMSE & MAXE \\
\cline { 2 - 5 } & Zenithal angle $\Delta \theta_{S}$ & $466.8^{\prime \prime}\left(0.13^{\circ}\right)$ & $516.8^{\prime \prime}\left(0.14^{\circ}\right)$ & $848.3^{\prime \prime}\left(0.24^{\circ}\right)$ \\
\cline { 2 - 5 } & Solar vector $\Delta V_{S}$ & $652.3^{\prime \prime}\left(0.18^{\circ}\right)$ & $674.5^{\prime \prime}\left(0.19^{\circ}\right)$ & $1101.3^{\prime \prime}\left(0.31^{\circ}\right)$ \\
\hline \multirow{2}{*}{ MICH } & Azimuth $\Delta \alpha_{S}$ & $-4.9^{\prime \prime}$ & $24.8^{\prime \prime}$ & $99.4^{\prime \prime}$ \\
\cline { 2 - 5 } $1980-2030$ & Zenithal angle $\Delta \theta_{S}$ & $7.9^{\prime \prime}$ & $26.0^{\prime \prime}$ & $60.1^{\prime \prime}$ \\
\cline { 2 - 5 } & Solar vector $\Delta V_{S}$ & $28.7^{\prime \prime}$ & $31.4^{\prime \prime}$ & $61.8^{\prime \prime}$ \\
\hline \multirow{2}{*}{ ENEA } & Azimuth $\Delta \alpha_{S}$ & $-0.0^{\prime \prime}$ & $2.9^{\prime \prime}$ & $10.6^{\prime \prime}$ \\
\cline { 2 - 5 } 2003-2022 & Zenithal angle $\Delta \theta_{S}$ & $-0.0^{\prime \prime}$ & $1.5^{\prime \prime}$ & $6.3^{\prime \prime}$ \\
\cline { 2 - 5 } & Solar vector $\Delta V_{S}$ & $2.3^{\prime \prime}$ & $2.7^{\prime \prime}$ & $8.0^{\prime \prime}$ \\
\hline
\end{tabular}

Table 1. Errors of three available fast solar position algorithms (SG, MICH, ENEA) for the computation of solar azimuth, solar zenithal angle, and solar vector at the geo-location $\left(45^{\circ} \mathrm{N}, 0^{\circ} \mathrm{E}\right)$, for 20000 instants during daylight randomly sampled in the period 1980 to 2030 (except for ENEA whose time period of validity is 2003-2022). The algorithm SPA is used as reference.

We observe in Table 1 and Fig. 1 that SG is less accurate than MICH by one order of magnitude for these three quantities. Performances of ENEA are excellent for the period 2003-2022, for which it has been optimized. ENEA is more accurate than MICH by one order of magnitude for this period; errors are very low. Fig. 1 shows that performances of ENEA are worse than MICH outside its time period of validity: prior to 2000 and after 2025; they are even worse than SG prior to 1995 . This illustrates the complexity of the trade-off between accuracy, covered period, complexity in implementation and computational speed. 


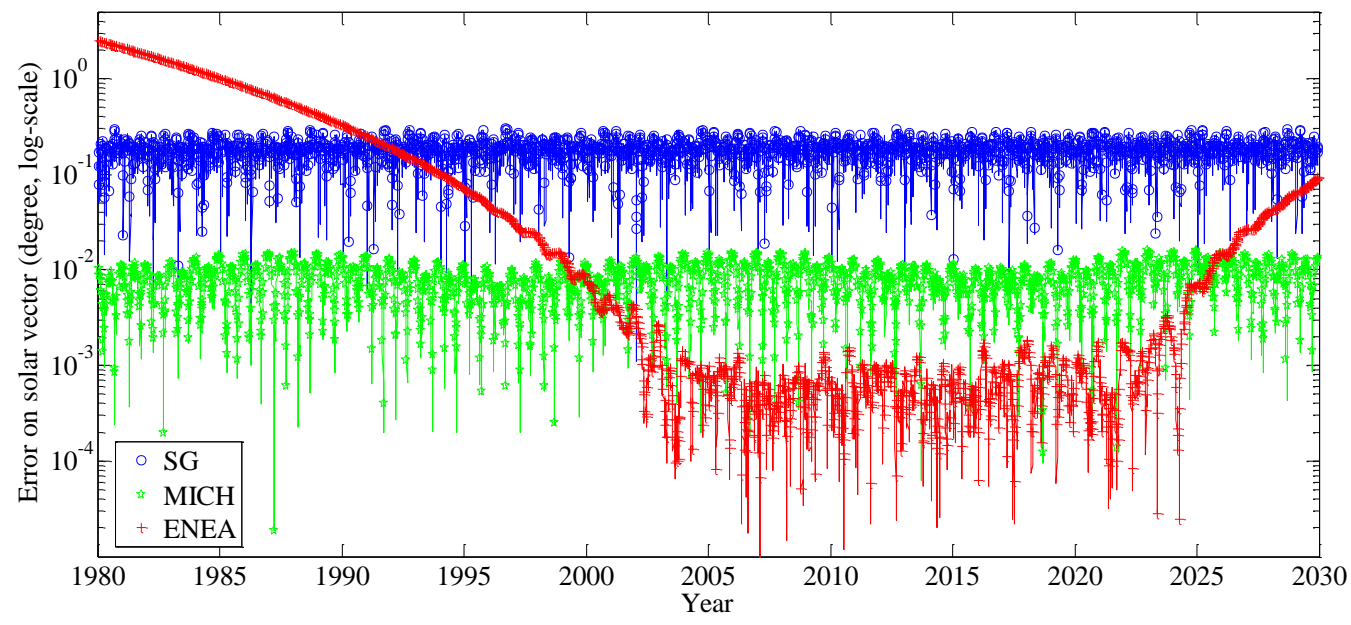

Figure 1. Errors on the solar vector predictions for the three fast algorithms (SG, MICH, and ENEA) during the reference time period 1980 to 2030, for the 20000 random sampled instants, at the geolocation $\left(45^{\circ} \mathrm{N}, 0^{\circ} \mathrm{E}\right)$.

We have also assessed the performances of each algorithm for computing time of sunrise and daily beam normal irradiation from beam horizontal irradiation, taking as reference the sun positions given by SPA. For this example, the computations were made arbitrarily for the date 2009-12-15, the longitude $0^{\circ}$ and three latitudes: $0^{\circ}, 45^{\circ} \mathrm{N}, 65^{\circ} \mathrm{N}$. Sampling time for computing sun positions and Riemann sums was $1 \mathrm{~s}$.

The sunrise predicted by a solar position algorithm, for a given day, corresponds to the minimum time when the Sun elevation angle is greater to 0 . Therefore, the error $\Delta t_{S R}$ for the time of sunrise predicted by the tested solar position algorithm $\mathrm{X}$ is defined by:

$$
\Delta t_{S R}=\min \left(\left\{t / \gamma_{S}^{X}(t)>0\right\}\right)-\min \left(\left\{t / \gamma_{S}^{S P A}(t)>0\right\}\right)
$$

where $\theta_{S}^{X}$ is the sun zenith angle predicted by the algorithm $\mathrm{X}$ and $\theta_{S}^{S P A}$ is the sun zenith angle predicted by the reference solar position algorithm SPA.

The direct or beam horizontal component $B$ of the irradiation at ground level is often assessed for the global horizontal irradiation using a solar processing splitting the global into direct and diffuse components (ESRA, 2000). In this example, we assume that the extinction $K$ of the beam irradiance and the normal irradiance at the top of atmosphere $E_{0}$ are both constant over the day:

$$
B(t)=E_{0} K \cos \theta_{s}^{S P A}(t)
$$

Of particular interest to thermodynamic or photovoltaic concentrated solar energy conversion systems is the beam component in normal incidence, noted $B_{N I}$ : 


$$
B_{N I}(t)=E_{0} K \Uparrow_{\cos \theta_{s}^{S P A}(t)>0}(t)
$$

where $\Uparrow_{C}(t)$ is the indicator function for the condition $\mathrm{C}(\mathrm{t})$.

When using the solar position algorithm $\mathrm{X}$, the beam normal irradiance from the beam component on horizontal surface is actually computed as follows:

$$
B_{N I}^{X}(t)=\frac{B(t)}{\cos \theta_{S}^{X}}=E_{0} K \frac{\cos \theta_{S}^{S P A}(t)}{\cos \theta_{S}^{X}(t)} \Uparrow_{\cos \theta_{s}^{X}(t)>0}(t)
$$

The relative error $\rho_{N I}$ in the daily beam normal irradiation is defined:

$$
\rho_{N I}=\frac{\int_{\cos \theta_{S}^{X}>0} B_{N I}^{X}(t) d t-\int_{\cos \theta_{S}^{S P A}>0} B_{N I}^{S P A}(t) d t}{\int_{\cos \theta_{S}^{S P A}>0} B_{N I}^{S P A}(t) d t}
$$

where the integrals are computed with Riemann sums with a sampling period of $1 \mathrm{~s}$.

We report in Table 2 the error $\Delta t_{S R}$ in astronomical sunrise as well the relative error $\rho_{N I}$ :

\begin{tabular}{cccc}
\hline \multirow{2}{*}{ Algorithm } & Latitude & $\Delta t_{S R}(\mathrm{~s})$ & $\rho_{N I}(\%)$ \\
\hline \multirow{2}{*}{ SG } & $0^{\circ}$ & 19 & 0.4 \\
\cline { 2 - 4 } $1980-2030$ & $45^{\circ}$ & -23 & -2.0 \\
\cline { 2 - 4 } & $65^{\circ}$ & -194 & -15.6 \\
\hline MICH & $0^{\circ}$ & -7 & 0.3 \\
\cline { 2 - 4 } $1980-2030$ & $45^{\circ}$ & -19 & -0.4 \\
\cline { 2 - 4 } ENEA & $65^{\circ}$ & -69 & -5.8 \\
\hline $2003-2022$ & $0^{\circ}$ & 0 & 0.0 \\
\cline { 2 - 4 } & $45^{\circ}$ & 0 & 0.0 \\
\hline
\end{tabular}

Table 2. Differences between SPA and each of the three algorithms for the astronomical sunrise time and the relative error in daily beam normal irradiation.

One can see that the errors presented in Table 2, for all solar position algorithms, increase with latitude. The magnitude of error depends on the algorithm. The errors for ENEA are close to zero: it is very accurate. Those for SG are large; an error larger than $1 \mathrm{~min}$ is observed on sunrise at the latitude 
$65^{\circ} \mathrm{N}$. Furthermore, the relative error due only to the Sun position can amount up to $15.6 \%$ in the case of computation of beam normal irradiation from beam horizontal irradiation.

At latitude $65^{\circ} \mathrm{N}, \mathrm{MICH}$ performs better than SG. Nevertheless, error in Sunrise may amount to $69 \mathrm{~s}$. Relative error can be greater than $5.8 \%$ for computation of daily beam normal irradiation. The figures indicated here are valid for the selected day only and are given for illustration; no conclusion should be drawn for other days.

Table 2 demonstrates that an accurate assessment of the position of the Sun is needed for the accurate assessment of the SSI. Together with Table 1, it also demonstrates that none of the proposed algorithms meets our requirements. After having contemplated the extension or enhancement of the three algorithms, we have opted for a new algorithm SG2, which is described now. The target for SG2 is the accuracy achieved by ENEA but extended to the period 1980-2030, instead of 2003-2022.

\section{TIME SYSTEMS}

Various time references should be considered. The International Atomic Time (TAI) is the basis of the definition of the second, under the responsibility of the Bureau International des Poids et Mesures (BIPM, www.bipm.org). The terrestrial time TT is equal to the TAI plus $32.184 \mathrm{~s}$, and is the time scale of ephemerides for Earth heliocentric position. The daily rotation of the Earth about itself determines daytime and nighttime. A day is the time duration for one rotation; a day is divided into $24 \mathrm{~h}$ of $60 \mathrm{~min}$ each as an average (mean solar day). The Universal Time (UT), also noted UT1, corresponds to a fraction of the mean solar day; it is equal to 0 at midnight for longitude $0^{\circ}$. This time is not plainly regular due to irregularities in Earth rotation (Markov et al., 2010; Mendes Cerveira et al., 2009). The standard time is Coordinated Universal Time, abbreviated UTC. It is the basis of legal time and is derived from International Atomic Time. The difference between UT and UTC is kept less than $1 \mathrm{~s}$. Therefore, the difference between UTC and TAI varies with the years and is under the responsibility of the International Earth Rotation Service (McCarthy, Petit, 2003), which issues every June and December a "Bulletin C" message (ftp://hpiers.obspm.fr/iers/bul/bulc/bulletinc.dat), which reports the introduction or not of a leap second, in order to guarantee $\mid$ UT-UTC $\mid<1$ s. Algorithms for Sun position, including the one presented in this paper, usually require UT: to avoid small but systematic angular errors related to the use of such algorithms in UTC time, one should refer to the "Bulletin D" message at ftp://hpiers.obspm.fr/iers/bul/buld to get updated (bulltetind.dat) and previous (bulletind.*) values of UT-UTC.

Of particular interest is the difference between the time TT and UT, $\Delta_{T T}$ :

$$
\Delta_{\mathrm{TT}}=\mathrm{TT}-\mathrm{UT}
$$


Values of $\Delta_{T T}$ are derived from observations only. The United States Naval Observatory (USNO) provides monthly values of $\Delta_{T T}$ updated every 4 months, and a prediction of quarterly values for the next seven years. The SPA also needs such values as an input parameter to correct for the Earth irregular rotation (Reda and Andreas, 2004).

Analytical approximation of $\Delta_{T T}$ offers advantage in implementation and operation of the algorithm and in prediction. Morrison and Stephenson (2004) proposed polynomial approximations for three different periods: 1961-1986, 1986-2005, 2005-2050. Espenak and Meeus (2009) brought corrections to these approximations. The last approximation was established in 2005 and has not yet been updated.

We propose to adopt these approximations whose piece-wise form per period permits to add new approximations to the existing ones to account for measures and 9-year predictions of $\Delta_{T T}$ issued every 4 months by the USNO respectively at the web addresses http://maia.usno.navy.mil/ser7/deltat.data and http://maia.usno.navy.mil/ser7/deltat.preds.

If $y$ denotes the year, in integer form, e.g., 2010, and $m$ the month ranging from 1 to 12 , the year $y$ in decimal form is:

$$
\mathbf{y}=y+(m-0.5) / 12
$$

We propose an approximation, notes $\Delta_{\mathrm{TT}-\mathrm{SG} 2}$ :

$$
\Delta_{I T}(y) \approx \Delta_{I T-S G 2}(\mathbf{y})=\left\{\begin{array}{l}
\sum_{k=0}^{5} a_{1, k}\left(y-y_{1}\right)^{k} \text { if } \mathrm{y} \in[1980,1986] \\
\sum_{k=0}^{5} a_{2, k}\left(y-y_{2}\right)^{k} \text { if } \mathrm{y} \in[1986,2005] \\
\sum_{k=0}^{3} a_{3, k}\left(y-y_{3}\right)^{k} \text { if } \mathrm{y} \in[2005,2030]
\end{array}\right.
$$

The Table 3 provides the parameters $\mathrm{y}_{\mathrm{i}}$ and $a_{i, k}$. These parameters are derived from Morrison and Stephenson (2005) including modifications proposed by Espenak and Meerus (2009). Parameters of the last third period 2005-2030 have been slightly modified to take into account USNO measurements up to May 2012. 


\begin{tabular}{lcccccccc}
\hline$n$ & Period & $y_{n}$ & $a_{n, 0}$ & $a_{n, 1}$ & $a_{n, 2}$ & $a_{n, 3}$ & $a_{n, 4}$ & $a_{n, 5}$ \\
\hline 1 & {$[1980,1986]$} & 1975 & 45.45 & 1.067 & $-1 / 260$ & $-1 / 718$ & - & - \\
\hline $2[1986,2005]$ & 2000 & 63.86 & 0.3345 & -0.060374 & 0.0017275 & $6.518 \times 10^{-4}$ & $2.374 \times 10^{-5}$ \\
\hline $3[2005,2030]$ & 2000 & 63.48 & 0.2040 & 0.005576 & - & - & - \\
\hline
\end{tabular}

Table 3. Parameters for $\Delta_{\mathrm{TT}-\mathrm{SG} 2}$.

In astronomy, it is convenient to use Julian days. The Universal Time Julian day $j_{U T}$ corresponds to the decimal number of days starting from January 1, in the year -4712 at 12:00:00 UT. It is computed from the UT time, whereas the Julian Ephemeris Day, or terrestrial Julian Day, $j_{T T}$, is computed from the terrestrial time TT. An algorithm to convert Julian days to current dates was described by Fliegel, Flandern (1968). A number of tools are available on the web or as basic functions in programming languages, e.g. php or Python, to convert dates into Julian days and conversely.

Assume a given instant with year $y$ greater than 1582, a month $m$, a day $d$, and a decimal UT decimal hour $h$. Then, let define interim quantities:

$$
\begin{aligned}
& y^{\prime}=y-1 \text { if } m=1 \text { or } 2, y^{\prime}=y \text { otherwise } \\
& m^{\prime}=m+12 \text { if } m=1 \text { or } 2, m^{\prime}=m \text { otherwise }
\end{aligned}
$$

then

$$
\begin{aligned}
j_{U T}= & \operatorname{INT}\left(365.25 y^{\prime}+4716\right)+\operatorname{INT}\left(30.6001\left(m^{\prime}+1\right)\right) \\
& +d+h / 24-I N T\left(y^{\prime} / 100\right)-1522.5
\end{aligned}
$$

where the function INT is the mathematical operator that rounds a decimal number to the nearest integers towards minus infinity (operator floor in $\mathrm{C}$ or Matlab).

The following relationship holds:

$$
j_{T T}=j_{U T}+\Delta_{\mathrm{TT}} / 86400
$$

For the sake of simplicity in the following, we will define $j_{U T}^{*}$, respectively $j_{T T}^{*}$, the Julian day defined as being 0 at 1980-01-01T00:00:00 respectively UT and TT:

$$
\begin{aligned}
& j_{U T}^{*}=j_{U T}-2444239.5 \\
& j_{T T}^{*}=j_{T T}-2444239.5
\end{aligned}
$$




\section{MAIN SUN PARAMETERS OF THE SUN FOR SOLAR ENERGY PURPOSES}

Let consider a local observer on Earth, located by its geographical latitude $\varphi$ (rad), longitude $\lambda$ (rad) and its elevation $\mathrm{h}(\mathrm{m})$ according to a given reference geographical ellipsoid. For this local observer, at the time $j_{U T}^{*}$, the Sun position can be defined by:

- the Sun topocentric elevation angle $\gamma_{\mathrm{s}}$ : the angle formed by the direction of the Sun and the horizon. Its complementary angle, the Sun topocentric zenith angle, is noted $\theta_{\mathrm{S}}$;

- the Sun topocentric azimuth angle $\alpha_{s}$ : the angle formed by the projection of the direction of the Sun on the horizontal plane defined eastward from North, following the ISO 19115 standard.

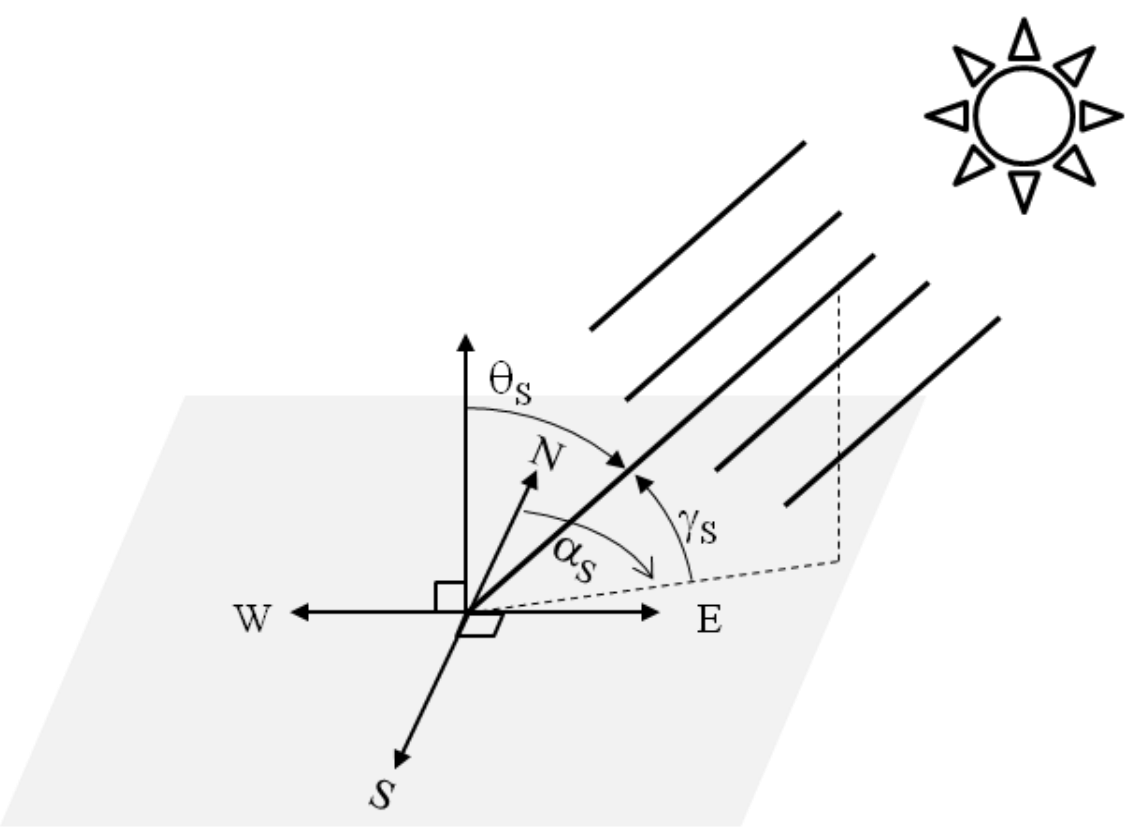

Figure 2. Sun topocentric elevation angle $\gamma_{\mathrm{S}}$, zenith angle $\theta_{\mathrm{S}}$ and azimuth angle $\alpha_{\mathrm{S}}$.

The cosine of the Sun incidence angle $\theta_{I}$ for a tilted plane whose orientation is defined by the surface azimuth angle $\alpha$ also measured eastward from North and its slope $\beta$ is given by:

$$
\cos \theta_{I}(\alpha, \beta)=\cos \theta_{S} \cos \beta+\sin \beta \sin \theta_{S} \cos \left(\alpha_{S}-\alpha\right)
$$

The most significant source of temporal variation of the broadband extraterrestrial irradiance on normal incidence $E_{0}$ is due to the temporal variation of the Earth-Sun distance. The broadband solar constant $E_{S C}$ is the average of $E_{0}$ over a year and corresponds to $E_{0}$ for a distance of $1 \mathrm{AU}$ :

$$
E_{0}=E_{S C} R^{-2}
$$

where $R$ is the Earth heliocentric radius expressed in AU and is discussed later. 
The value of the broadband solar constant $\mathrm{E}_{\mathrm{sc}}$ is set to $1367 \mathrm{~W} / \mathrm{m}^{2}$ : the typical day-to-day relative variations of $\mathrm{E}_{\mathrm{SC}}$ due to solar activity are less than $0.15 \%$ and are considered negligible in the field of solar energy (Schatten and Orosz, 1990; Wald, 2007).

These Sun parameters $\gamma_{S}, \theta_{S}, \alpha_{S}, \cos \theta_{I}$ and $E_{0}$ are commonly used for solar energy studies as they enable for example the computations of the extraterrestrial surface solar irradiance on a tilted plan, the sunrise and sunset times or the shadow effects due to local horizon. 


\section{COMPUTATION PROCEDURE}

The main Sun parameters $\gamma_{S}, \cos \theta_{S}, \alpha_{S}, \theta_{I}$ and $E_{0}$ are computed following the sequence of equations given by the Table 4 .

1. Heliocentric coordinates

\begin{tabular}{lll} 
Sun heliocentric radius & $R$ & see sub-section 6.1, Eq. (25) \\
\hline Sun heliocentric longitude & $L$ & see sub-section 6.1, Eq. (26)
\end{tabular}

2. Geocentric parameters

Stellar aberration correction

Sun equatorial horizontal parallax

Nutation in the Sun geocentric longitude

True Earth obliquity

Apparent Sun geocentric longitude

Sun Geocentric declination

Sun Geocentric right ascension

$$
\begin{array}{ll}
\Delta \tau & \text { see sub-section 6.2, Eq. (27) } \\
\xi & \text { see sub-section 6.2, Eq. (28) } \\
\Delta \psi & \text { see sub-section 6.2, Eq. (29) } \\
\varepsilon & \text { see sub-section 6.2, Eq. (30) } \\
\Theta_{a} & L+\pi+\Delta \psi+\Delta \tau \\
\delta^{g} & \operatorname{asin}\left(\sin \Theta_{a} \sin \varepsilon\right) \\
r_{\alpha}^{g} & \operatorname{atan} 2\left(\sin \Theta_{a} \cos \varepsilon, \cos \Theta_{a}\right)
\end{array}
$$

\section{Sidereal time}

Mean sidereal time

$v_{0} \quad$ see sub-section 6.4, Eq. (31)

4. Topocentric parameters

Parallax effects in the sun topocentric right ascension

Sun topocentric declination

$\Delta r_{\alpha} \quad$ see sub-section 6.3, eq. (33)

Sun topocentric hour angle

$\delta \quad$ see sub-section 6.3, eq. (35)

Sun topocentric azimuth

$\omega \quad v_{0}+\Delta \psi \cos \varepsilon-r_{\alpha}^{g}+\lambda-\Delta r_{\alpha}$

$\alpha_{S} \quad \operatorname{atan} 2(\sin \omega, \cos \omega \sin \varphi-\tan \delta \cos \varphi)+\pi$

Sun topocentric elevation angle without atmospheric refraction

$\gamma_{S O} \quad \operatorname{asin}(\sin \varphi \sin \delta+\cos \varphi \cos \delta \cos \omega)$ correction

Atmospheric refraction correction term $\Delta \gamma_{S}$

Sun topocentric elevation angle

$$
\gamma_{S} \quad \gamma_{S 0}+\Delta \gamma_{S}
$$

Table 4. Formulas used for the computation procedure.

These equations come from Reda and Andreas (2004, corrigendum 2007), except for:

- the Earth heliocentric radius $R$ and longitude $L$, the true Earth obliquity $\varepsilon$ the nutation in the Sun geocentric longitude $\Delta \psi$ and the mean sidereal time $v_{0}$ for which approximations based on 1-order polynomial and truncated sinusoidal series are proposed. 
- the Sun equatorial horizontal parallax $\xi$ and the stellar aberration correction $\Delta \tau$ for which constant values are proposed ;

- the parallax effects in the sun topocentric right ascension $\Delta r_{\alpha}$ and Sun topocentric declination $\delta$ for which approximations based on simple 1-order Taylor expansion are proposed ;

- the atmospheric refraction correction $\Delta \gamma_{s}$ for which we propose to use an extended version for low negative Sun topocentric elevation angle ;

\subsection{Heliocentric coordinates $R$ and $L$}

We have used sinusoidal series to approximate the Earth heliocentric coordinates $R$ and $L$ on the reference period 1980-2030. The parametric function for these sinusoidal approximations is made up of an affine function defined by two parameters $a$ and $b$, and $N$ sinus functions, each defined by a frequency $f_{k}$, a phase $\phi_{k}$ and a module $\rho_{k}$ :

$$
\sum_{k=1}^{N} \rho_{k} \cos \left(2 \pi f_{k} j_{T T}^{*}-\phi_{k}\right)+a j_{T T}^{*}+b
$$

For each quantities to approximate, the frequencies $f_{k}$ are first determined by a Fourier analysis of the discrete vector calculated with SPA on the reference period 1980-2030, with a time sampling of 1 hour (i.e. 447072 discrete values). This analysis consists in sorting, in descending order, the complex modulus of local maxima of the Fourier coefficients, for positive frequencies. Then the first $N$ corresponding frequencies are selected. The number $N$ of selected frequencies depends on the level of required precision of the approximation. Once these $N$ frequencies are selected, the $2 N+2$ parameters $a, b, \rho_{1} \cos \phi_{1}, \rho_{1} \sin \phi_{1}, \ldots, \rho_{N} \cos \phi_{N}$ and $\rho_{N} \sin \phi_{N}$ of Eq. (23) are determined by a linear mean square regression. All these parameters, including the frequencies, are then refined by the LevensbergMarquardt non-linear least-square method (Marquardt, 1963).

\section{Approximation of the Earth heliocentric radius $R$}

The approximation of the Earth heliocentric radius $R$ should be accurate enough to be able to predict the eccentricity correction term with a relative accuracy $\eta$ better than $0.1 \%$. This accuracy threshold comes from the negligible order of magnitude due to solar activity. The 0 -order Taylor-Lagrange formula applied to the eccentricity correction term shows that the absolute value of the Earth heliocentric radius error $\delta R$ should observe the following inequality relationship:

$$
|\delta R|<\eta \frac{\min (R)^{3}}{2 \max (R)^{2}} \approx 4.60 \times 10^{-4} \mathrm{AU}
$$


With $N=1$ and the parameters shown in Table 5, the approximation of the Earth heliocentric radius $\mathrm{R}_{\mathrm{SG} 2}$ is better than $2.3 \times 10^{-4} \mathrm{AU}$ for the reference time period:

$$
R \approx R_{S G 2}=\rho^{R} \cos \left(2 \pi f^{R} j_{T T}^{*}-\phi^{R}\right)+a^{R} j_{T T}^{*}+b^{R}
$$

\begin{tabular}{cc}
\hline$a^{R}$ & $b^{R}$ \\
\hline 0 & 1.000140
\end{tabular}

\begin{tabular}{ccc}
\hline$f^{R}$ & $\rho^{R}$ & $\phi^{R}$ \\
\hline $1 / 365.254902$ & 0.016704 & -3.091159
\end{tabular}

Table 5. Parameters for the approximation $\mathrm{R}_{\mathrm{SG} 2}$ of the Earth heliocentric radius, for the reference period 1980-2030.

Although this approximation $\mathrm{R}_{\mathrm{SG} 2}$ is fast to compute with only one sinus function, we have studied the possibility of using a pre-computed sampled table of $\mathrm{R}_{\mathrm{SG} 2}$, combined with nearest or linear interpolation between the temporal nodes of the table.

Upper bounds of nearest or linear interpolation errors, computed using equations A2, A3 in annex, have assessed the errors yielded by both interpolations when applied to a sampled pre-computed table of $R_{S G 2}$, for different time sampling expressed in number of days. Considering the required overall accuracy of Earth heliocentric radius prediction and the accuracy of the approximation function $R_{S G 2}$ itself, the interpolation should not yield errors greater than $2.3 \times 10^{-4} \mathrm{AU}$.

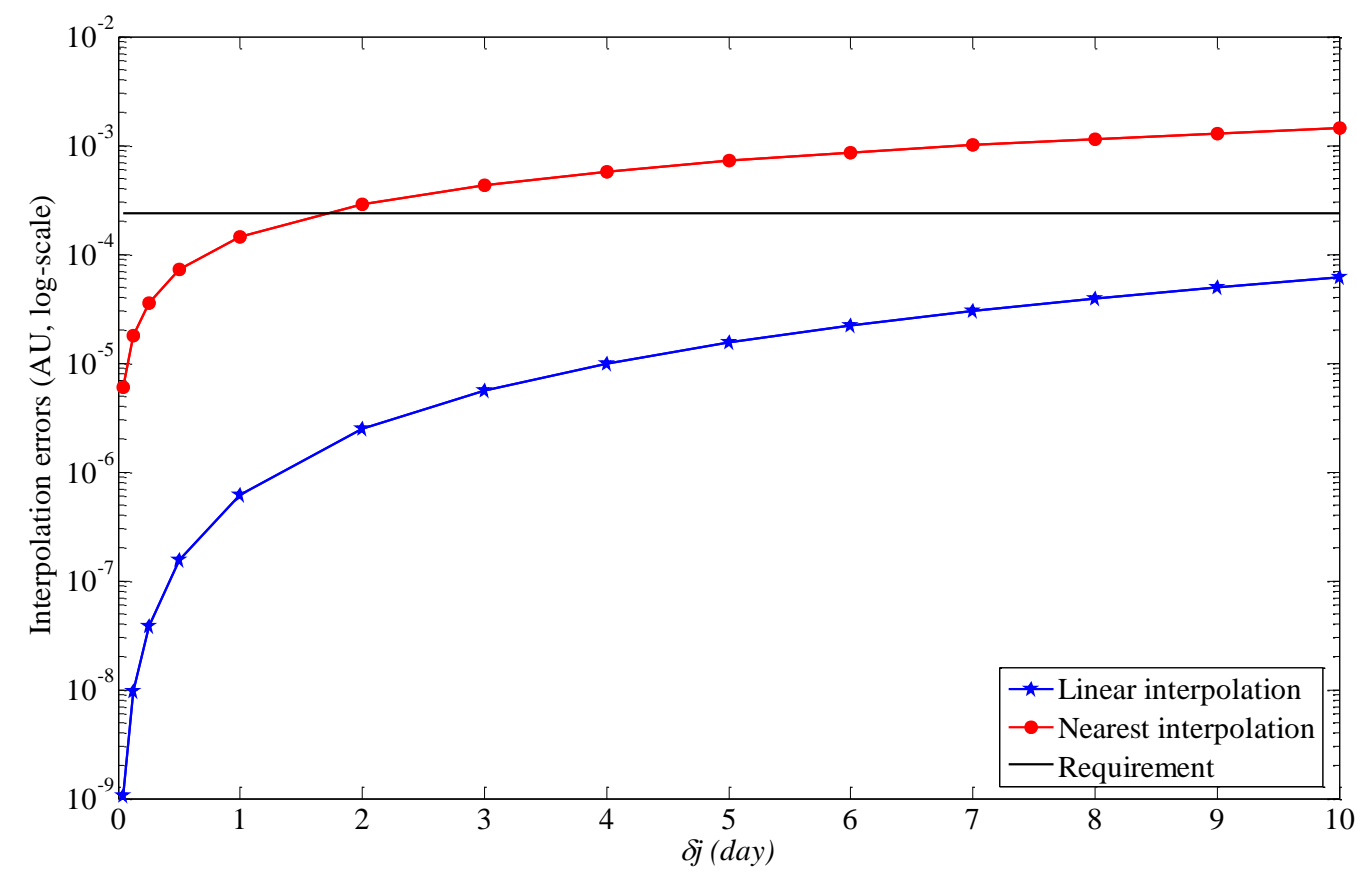

Figure 3. Upper bounds of nearest $(\bullet)$ and linear ( $\star$ interpolation errors of $R_{S G 2}$ for different time sampling time $\delta j$, compared to the accuracy target (green) of $2.3 \mathrm{e}-4 \mathrm{AU}$. 
Fig. 3 depicts the upper bounds of nearest and linear interpolation errors of $R_{S G 2}$ for different time sampling. Linear interpolation always fits the accuracy requirement even with a pre-computed table of $R_{S G 2}$ sampled every 10 days. As for the nearest interpolation, at least to a daily pre-computed table should be sampled to fit the accuracy requirement.

Nearest interpolation applied on daily pre-computed table of $R_{S G 2}$ has been finally chosen for SG2, as it requires only 2 floating operations per interpolation request, instead of 7 floating operations for the linear interpolation.

\section{Approximation of the Earth heliocentric longitude $L$}

In order to determine the required overall accuracy of the approximation, we have analysed the performance of the ENEA algorithm for the prediction of the Earth heliocentric longitude $L$. Over the period of validity of this algorithm, the root mean square of the prediction is $2.5^{\prime \prime}$ with a maximum of absolute error of $8 "$. In order to limit the number of sinus functions, we have chosen, for the reference period, an accuracy target of 10" for the maximum absolute error and 3 " for the root mean square error. The minimum number of sinus functions fitting this requirement is 10 . The 32 parameters of this approximation functions $L_{S G 2}$ are given Table 6:

\begin{tabular}{|c|c|c|c|c|c|}
\hline$a^{L}$ & $b^{L}$ & $k$ & $f_{k}^{L}$ & $\rho_{k}^{L}$ & $\phi_{k}^{L}$ \\
\hline \multirow[t]{10}{*}{$1 / 58.130101$} & 1.742145 & 1 & $1 / 365.261278$ & $3.401508 \times 10^{-2}$ & 1.600780 \\
\hline & & 2 & $1 / 182.632412$ & $3.486440 \times 10^{-4}$ & 1.662976 \\
\hline & & 3 & $1 / 29.530634$ & $3.136227 \times 10^{-5}$ & -1.195905 \\
\hline & & 4 & $1 / 399.529850$ & $3.578979 \times 10^{-5}$ & -1.042052 \\
\hline & & 5 & $1 / 291.956812$ & $2.676185 \times 10^{-5}$ & 2.012613 \\
\hline & & 6 & $1 / 583.598201$ & $2.333925 \times 10^{-5}$ & -2.867714 \\
\hline & & 7 & $1 / 4652.629372$ & $1.221214 \times 10^{-5}$ & 1.225038 \\
\hline & & 8 & $1 / 1450.236684$ & $1.217941 \times 10^{-5}$ & -0.828601 \\
\hline & & 9 & $1 / 199.459709$ & $1.343914 \times 10^{-5}$ & -3.108253 \\
\hline & & 10 & $1 / 365.355291$ & $8.499475 \times 10^{-4}$ & -2.353709 \\
\hline
\end{tabular}

Table 6. Parameters for the approximation $\mathrm{L}_{\mathrm{SG} 2}$ of the Earth heliocentric longitude with Eq. (36), for the reference period 1980-2030.

The maximum absolute error of the prediction $L_{S G 2}$ of $L$ is circa $9 "$ ". The analysis of the upper bound of interpolation errors shows that linear interpolation of daily pre-computed table of $L_{S G 2}$ yields absolute 
errors less than 1". Therefore, linear interpolation applied on daily pre-computed table of $L_{S G 2}$ has been finally chosen for the Earth heliocentric longitude in SG2.

\subsection{Geocentric parameters $\Delta \tau, \xi, \Delta \psi$ and $\varepsilon$}

The Sun equatorial horizontal parallax $\xi$ and the stellar aberration correction $\Delta \tau$ are inversely proportional to the Earth heliocentric radius. Following Grena (2008), we have chosen constant approximations of these correction terms, leading to negligible absolute errors, less than $0.4 "$ :

$$
\begin{aligned}
& \Delta \tau \approx \Delta \tau_{S G 2}=-9.933735 \times 10^{-5} \mathrm{rad} \\
& \xi \approx \xi_{S G 2}=4.263521 \times 10^{-5} \mathrm{rad}
\end{aligned}
$$

Following the same methodology used for the approximations of the heliocentric coordinates $R$ and $L$, the nutation in the Sun geocentric longitude $\Delta \psi$ and the true Earth obliquity $\varepsilon$ and can be approximated by the two followings sinusoidal functions, with parameters given in Tables 7 and 8:

$$
\Delta \psi \approx \Delta \psi_{S G 2}=\rho^{\Delta \psi} \cos \left(2 \pi f^{\Delta \psi} j_{T T}^{*}-\phi^{\Delta \psi}\right)
$$

\begin{tabular}{ccc}
\hline$f^{\Delta \psi}$ & $\rho^{\Delta \psi}$ & $\phi^{\Delta \psi}$ \\
\hline $1 / 6791.164405$ & $8.329092 \times 10^{-5}$ & -2.052757
\end{tabular}

Table 7. Parameters for the approximation $\Delta \psi_{S G 2}$ of the nutation in the Sun geocentric longitude for the reference period 1980-2030.

$$
\varepsilon \approx \varepsilon_{S G 2}=\rho^{\varepsilon} \cos \left(2 \pi f^{\varepsilon} j_{T T}^{*}-\phi^{\varepsilon}\right)+a^{\varepsilon} j_{T T}^{*}+b^{\varepsilon}
$$

\begin{tabular}{cc}
\hline$a^{\varepsilon}$ & $b^{\varepsilon}$ \\
\hline$-6.216374 \times 10^{-9}$ & $4.091383 \times 10^{-1}$
\end{tabular}

\begin{tabular}{ccc}
\hline$f^{\varepsilon}$ & $\rho^{\varepsilon}$ & $\phi^{\varepsilon}$ \\
\hline $1 / 6791.164405$ & $4.456183 \times 10^{-5}$ & 2.660352
\end{tabular}

Table 8. Parameters for the approximation $\overline{\varepsilon_{S G 2} \text { of the true Earth obliquity for the reference period }}$ 1980-2030.

The errors in prediction of true Earth obliquity and the nutation in Sun geocentric longitude provided respectively by $\varepsilon_{S G 2}$ and $\Delta \psi_{S G 2}$ are both less than 2", including nearest interpolations of daily precomputed tables.

\subsection{Sidereal time $v_{0}$}


Reda and Andreas (2004) proposed a formulation of the mean sidereal time at Greenwich $v_{0}$ based on a quintic function of universal Julian days. On the reference time period 1980-2030, a simple linear model is sufficient to provide an approximation with absolute errors less than 0.1 ":

$$
v_{0} \approx v_{0-S G 2}=6.3000388 j_{U T}^{*}+1.742079
$$

\subsection{Topocentric coordinates: approximations of $\Delta r_{\alpha}, \delta$ and $\Delta \gamma_{s}$}

Let consider the following intermediate parameters related to the geographical position of the observer and the reference geographical ellipsoid:

$$
\begin{aligned}
& u=\operatorname{atan}((1-f) \tan \varphi) \\
& x=\cos u+\frac{h}{a} \cos \varphi \\
& y=(1-f) \sin u+\frac{h}{a} \sin \varphi
\end{aligned}
$$

The reference geographical ellipsoid is generally described by the data of two of the following three parameters: the semi-major axis $a$, semi-minor axis $b$ and flattening $f$. Table 9 gives the parameters used by Reda and Andreas (2004):

\begin{tabular}{rcc}
\hline$a(\mathrm{~m})$ & $b(\mathrm{~m})$ & $1 / f$ \\
\hline 6378140.0 & 6357014.436696 & 298.257282697
\end{tabular}

Table 9. Parameters of the reference geographical ellipsoid used by Reda and Andreas (2004).

Reda and Andreas (2004) proposed a formula to $\Delta r_{\alpha}$ with a call to the inverse trigonometric function atan2. By using the 1-order Taylor expansion of this formula with respect to the small value of $\Delta r_{\alpha}$ $(\sim 8 ")$ we avoid this call:

$$
\Delta r_{\alpha} \approx \Delta r_{\alpha-S G 2}=-x \frac{\sin \omega^{g}}{\cos \delta^{g}} \xi
$$

Grena (2008) proposed another approximation that leads to absolute errors less than $0.9 "$, whereas the proposed 1-order Taylor expansion leads to absolute errors less than $0.15 "$.

From Reda and Andreas (2004, corrigendum 2007), the Sun topocentric declination $\delta$ is given by:

$$
\delta=\operatorname{atan} 2\left(\left(\sin \delta^{g}-y \sin \xi\right) \cos \Delta r_{\alpha}, \cos \delta^{g}-x \sin \xi \cos \omega^{g}\right)
$$

The 1-order Taylor expansion of Eq. (34) with respect to the small values of the parallax correction $\Delta r_{\alpha}$ and the Sun equatorial horizontal parallax $\xi$ yields the following approximation:

$$
\delta \approx \delta_{S G 2}=\delta^{g}+\left(x \cos \omega^{g} \sin \delta^{g}-y \cos \delta^{g}\right) \xi
$$


As for the parallax effects in the sun topocentric right ascension, Grena (2008) proposed another approximation that exhibits absolute errors less than 2.8 " whereas this 1-order Taylor expansion yields absolute errors less than 0.15 ", with the same order of computational complexity.

Reda and Andreas (2004) use the atmospheric refraction correction term of Meeus (1999), taking into account the yearly average local air pressure $P$ expressed in $\mathrm{hPa}$, the yearly average of local air temperature $\mathrm{T}$ expressed in ${ }^{\circ} \mathrm{C}$ and the Sun topocentric elevation angle.

This correction, valid for $\gamma_{\mathrm{s} 0}$ greater than $-0.01 \mathrm{rad}$, is

$$
\Delta \gamma_{S} \underset{\gamma_{S 0} \geq-0.01}{\approx} \Delta \gamma_{S-S G 2}^{+}=\frac{P}{1010} \frac{283}{273+T} \frac{2.96706 \times 10^{-4}}{\tan \left(\gamma_{S 0}+0.0031376\left(\gamma_{S 0}+0.089186\right)^{-1}\right)}
$$

For $\gamma_{\mathrm{S} 0}$ less than $-0.01 \mathrm{rad}$, we use the correction term of Cornwall et al. (2011):

$$
\Delta \gamma_{S} \underset{\gamma_{S 0<-0.01}}{\approx} \Delta \gamma_{S-S G 2}^{-}=-\frac{P}{1010} \frac{283}{273+T} \frac{1.005516 \times 10^{-4}}{\tan \gamma_{S 0}}
$$

\section{PERFORMANCES OF THE SG2 ALGORITHM}

To assess the performances in accuracy and precision of the proposed fast algorithm SG2 in the time period 1980-2030, we have done the same test procedure as described in the section $\S 3$ for the already existing fast solar position algorithms, with the same randomly sampled 20000 instants, between 1980 and 2030, for daylight time, at location $45^{\circ} \mathrm{N}$ and longitude $0^{\circ}$, at sea level. Results are reported in Table 10 and Fig. 4.

\begin{tabular}{ccccc}
\hline \multirow{2}{*}{ Algorithm } & Errors & MBE & RMSE & MAXE \\
\hline \multirow{2}{*}{ SG2 } & Azimuth $\Delta \alpha_{S}$ & $0.0^{\prime \prime}$ & $3.5^{\prime \prime}$ & $18.1^{\prime \prime}$ \\
\cline { 2 - 5 } $1980-2030$ & Zenithal angle $\Delta \theta_{S}$ & $-0.0^{\prime \prime}$ & $1.6^{\prime \prime}$ & $6.9^{\prime \prime}$ \\
\cline { 2 - 5 } & Solar vector $\Delta V_{S}$ & $2.4^{\prime \prime}$ & $2.8^{\prime \prime}$ & $8.9^{\prime \prime}$
\end{tabular}

Table 10. Errors for the computation with SG2 of the solar azimuth, the solar zenithal angle, and the solar vector (SPA is the reference) at the geo-location $\left(45^{\circ} \mathrm{N}, 0^{\circ} \mathrm{E}\right)$, for the same 20000 instants during daylight randomly sampled in the period 1980-2030 as Table 1. 


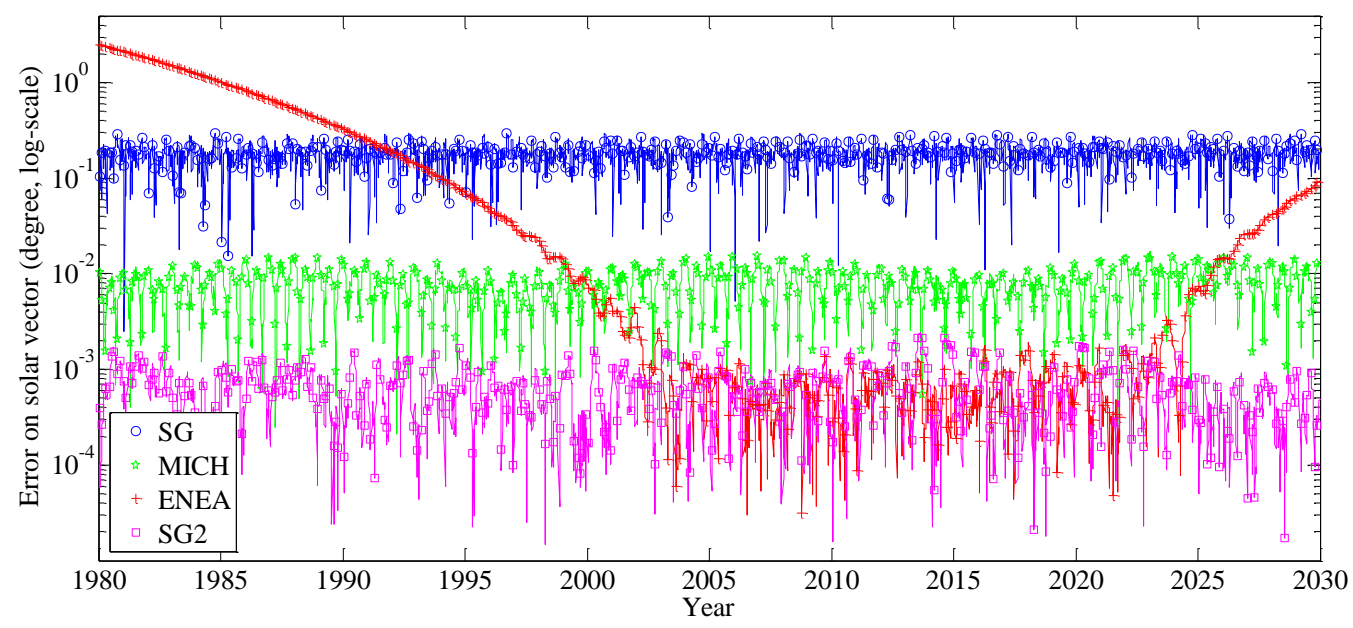

Figure 4. Errors on the solar vector predictions for the three fast algorithms (SG, MICH, and ENEA), compared to SG2, during the reference time period 1980-2030.

Compared to Table 1 and Fig. 1, we observe in Table 10 and Fig. 4 that accuracy performances provided by SG2 in the time period 1980-2030 is close to those provided by ENEA (Grena, 2008) but only for its restricted time period of validity 2003-2022.

In addition, without pre-computed tables of $L, R, \Delta \psi$ and $\varepsilon$, the total numbers of additions (40), multiplications (46) and calls for direct (32) and inverse trigonometric functions (6) needed to calculate Sun topocentric position are slightly greater than those needed by the ENEA algorithm. The approximations with sinusoidal series of $L, R, \Delta \psi$ and $\varepsilon$ enable the use of linear or nearest interpolations from pre-computed tables of these parameters with a controlled level uncertainty, thanks to the Eqs. (A3) and (A3). In this case, the total numbers of additions (20), multiplications (30) and calls for direct (17) and inverse trigonometric functions (6) needed to calculate Sun topocentric position are slightly less than those needed by the ENEA algorithm.

Between the submission and the revision dates of this paper, Grena (2012) has published an article presenting five new algorithms for the computation of the sun position from 2010 to 2110, with different levels of accuracy and computational complexity. Over this period of 100 years, the most accurate algorithm (algorithm 5) provides topocentric sun elevation and azimuth angles with root mean square errors of respectively 1.8 " and $4.2 "$ ", with a computational complexity close to that of SG2. When extended to the 100-year period from 1980 to 2080, SG2 offers similar performances, with root mean square errors of 2.3" and 4.2" respectively for the topocentric sun elevation and azimuth angles.

The time needed by SG2 for the computation of the solar positions every seconds within 15 min for the 9 millions usable pixels in a Meteosat SEVIRI image is less than $8 \mathrm{~min}$, compared to the $2 \mathrm{~h}$ needed for SPA and to the 8 min needed for SG. For a given instant, optimization can be also done by 
pre-computing tables for quantities not depending on the geographical coordinates, such as the sun geocentric positions.

\section{CONCLUSION}

The present article proposes a new algorithm SG2 which is fast and accurate: over the period 19802030 , the maximum error in the solar vector is less than $0.0025^{\circ}\left(\sim 9^{\prime \prime}\right)$ and the uncertainty of the Sun topocentric azimuth and elevation is $c .0 .0015^{\circ}\left(\sim 5^{\prime \prime}\right)$. It is accurate enough for calibration of pyranometers and pointing devices (Reda and Andreas, 2003, 2004; Stafford et al., 2009). It is fast enough for its use in the HelioClim processing and post-processing chains and other similar computational chains. It is accurate enough so that the influence of the Sun position on the error budget of satellite-derived assessment of SSI is of second-order or negligible.

Several approximations are made of the original equations of the SPA to decrease the number of operations. These approximations result in a decrease of accuracy compared to SPA and we have presented the mathematical tools permitting to determine these approximations with a selected uncertainty level. These tools will permit further development of other algorithms meeting other requirements than HelioClim. Like the ESRA algorithm, software libraries in C and Matlab are available on the Web (www.helioclim.org) and should help such developments. A Web service is also available at www.webservice-energy.org. It is an application that can be invoked via the Web. It could be used to check the correctness of implementation of the SG2 algorithm or can be used for the computation of the Sun position.

\section{ANNEX A - COMPUTATION OF UPPER BOUND OF ERROR IN INTERPOLATING A DISCRETE SINUSOIDAL SERIES}

Let consider a function $s(t)$ defined by a truncated Fourier series and an affine function:

$$
s(t)=\sum_{k=1}^{N} \rho_{k} \cos \left(2 \pi f_{k} t-\phi_{k}\right)+\alpha t+\beta
$$

$s_{\delta t}$ is a discrete signal resulting from the sampling of $s(t)$ with a sampling period $\delta t$, and an arbitrary sampling phase $t_{0}$ :

$$
s_{\delta t}(k)=s\left(k \delta t+t_{0}\right)
$$

The upper bounds of error when interpolating the discrete signal $s_{\delta t}$ by the nearest neighbor and linear interpolations are respectively noted $\mathrm{UB}_{\mathrm{N}}$ and $\mathrm{UB}_{\mathrm{L}}$, and are given: 


$$
\begin{aligned}
& U B_{N}=\left(\pi \sum_{k=1}^{N} \rho_{k} f_{k}+\frac{|\alpha|}{2}\right) \delta t \\
& U B_{L}=4 \pi^{2} \delta t^{2} \sum_{k=1}^{N} \rho_{k} f_{k}^{2}
\end{aligned}
$$

\section{ACKNOWLEDGMENTS}

The research leading to these results has received funding from the European Union's Seventh Framework Programme (FP7/2007-2013) under Grant Agreement no. 262892 (ENDORSE project). 


\section{REFERENCES}

Blanc, Ph., Gschwind, B., Lefèvre, M., Wald, L., 2011. The HelioClim project: surface solar irradiance data for climate application. Remote Sensing, in press, 3(2), 343-361, doi:10.3390/rs20x000x

Blanco-Muriel, M., Alarcón-Padilla, D. C., López-Moratalla, T., Lara-Coira, M., 2001. Computing the solar vector. Solar Energy, 70(5), 431-441.

Bretagnon, P., Francou, G., 1988. Planetary theories in rectangular and spherical variables. VSOP87 solutions. Astronomy \& Astrophysics, 202, 309-315.

Cornwall, C., Horiuchi, A., Lehman, C. 2011. Solar calculator. NOAA Earth System Research Lab. http://www.srrb.noaa.gov/highlights/sunrise/azel.html, last accessed: May 2011.

Cros, S., Albuisson, M., Lefèvre, M., Rigollier, C., Wald, L., 2004. HelioClim: a long-term database on solar radiation for Europe and Africa. In Proceedings of EuroSun 2004, published by PSE GmbH, Freiburg, Germany, pp. (3)916-920.

Diabaté, L., Moussu, G., Wald, L., 1989. Description of an operational tool for determining global solar radiation at ground using geostationary satellite images. Solar Energy, 42(3), 201-207.

Espenak, F., Meeus, J., 2009. Five millennium catalog of solar eclipses: -1999 to +3000 (2000 bce to 3000 ce)—revised. Technical Report NASA/TP-2009-214174, NASA.

Espinar, B., Ramírez, L., Polo, J., Zarzalejo, L.F., Wald, L., 2009. Analysis of the influences of uncertainties in input variables on the outcomes of the Heliosat-2 method. Solar Energy, 83, 17311741, doi:10.1016/j.solener.2009.06.010

ESRA, 2000. European Solar Radiation Atlas. Fourth edition, includ. CD-ROM. Edited by Greif, J., and K. Scharmer. Scientific advisors: R. Dogniaux, J. K. Page. Authors: L. Wald, M. Albuisson, G. Czeplak, B. Bourges, R. Aguiar, H. Lund, A. Joukoff, U. Terzenbach, H. G. Beyer, E. P. Borisenko. Published for the Commission of the European Communities by Presses de l'Ecole, Ecole des Mines de Paris, Paris, France.

Fliegel, H. F., Flandern, T. C. V., 1968. A machine algorithm for processing calendar dates. Communications of the ACM, 11(10), 657.

Grena, R., 2008. An algorithm for the computation of the solar position. Solar Energy, 82(5), 462-470, doi:10.1016/j.solener.2007.10.001.

R. Grena, 2012. Five new algorithms for the computation of sun position from 2010 to 2110, Solar Energy, vol. 86(5), 1323-1337, doi:10.1016/j.solener.2012.01.024. 
Gschwind, B., Ménard, L., Albuisson, M., Wald, L., 2006. Converting a successful research project into a sustainable service: the case of the SoDa Web service. Environmental Modelling and Software, 21, 1555-1561, doi:10.1016/j.envsoft.2006.05.002.

ISO 19115, 2003. Geographic information - Metadata. Correction 2006. International Organization for Standardization. Geneva, Switzerland.

Kambezidis, H. D., Tsangrassoulis, A. E., 1993. Solar position and right ascension. Solar Energy, $50(5), 415-416$.

Lefèvre, M., Diabaté, L., Wald, L., 2007. Using reduced data sets ISCCP-B2 from the Meteosat satellites to assess surface solar irradiance. Solar Energy, 81, 240-253, doi:10.1016/j.solener.2006.03.008.

Markov, Y. G., Perepelkin, V. V., Sinitsyn, I. N., Semendyaev, N. N., 2010. Rotational-oscillatory motion of the Earth and the global component of the seismic process. Doklady Physics, 55(11), 583587, doi: 10.1134/S1028335810110121.

McCarthy, D. D., Petit, G., 2003. IERS conventions (2003). IERS Technical Note 32, International Earth Rotation and Reference System Service. Verlag des Bundesamts für Kartographie und Geodäsie, Frankfurt am Main, Germany, 2004. 127 pp.

Marquardt, D., 1963. An algorithm for least-squares estimation of nonlinear parameters, SIAM Journal Applied Math., 11, 431-441.

Meeus, J., 1999. Astronomical Algorithms (2 ${ }^{\text {nd }}$ edition). Willmann-Bell Inc., Richmond, Va, USA, $477 \mathrm{pp}$.

Mendes Cerveira, P. J., Boehm, J., Schuh, H., Kluegel, T., Velikoseltsev, A., Schreiber, K. U., Brzezinski, A., 2009. Earth rotation observed by very long baseline interferometry and ring laser. Pure and Applied Geophysics, 166(8-9), 1499-1517, doi: 10.1007/s00024-004-0487-z.

Michalsky, J., 1988. The astronomical almanac's algorithm for approximate solar position (19502050). Solar Energy, 40(3), 227-235.

Morrison, L., Stephenson, F. R., 2004. Historical values of the Earth's clock error Delta T and the calculation of eclipses. Journal for the History of Astronomy, 35(120), 327-336.

Perez, R., Ineichen, P., Moore, K., Kmiecik, M., Chain, C., George, R., Vignola, F., 2002. A new operational model for satellite-derived irradiances: description and validation. Solar Energy, 73(5), 307-317, doi: 10.1016/S0038-092X(02)00122-6.

Pitman, C.L., Vant-Hull, L.L., 1978. Errors in locating the Sun and their effect on solar intensity predictions. In: Meeting of the American Section of the International Solar Energy Society, Denver, 28 August 1978, pp. 701-706. 
Reda, I., Andreas, A., 2003. Solar position algorithm for solar radiation applications: Technical Report, National Renewable Energy Laboratory, Golden, Co, USA. Revised version: January 2008.

Reda, I., Andreas, A., 2004. Solar position algorithm for solar radiation applications: Solar Energy, 76(5), 577-589. Corrigendum, 81, 838-838, 2007.

Rigollier, C., Lefèvre, M., Wald, L., 2004. The method Heliosat-2 for deriving shortwave solar radiation from satellite images. Solar Energy, 77(2), 159-169.

Stafford, B., Davis, M., Chambers, J., Martínez, M., Sanchez, D., 2009. Tracker accuracy: field experience, analysis, and correlation with meteorological conditions. In Proceedings of the $34^{\text {th }}$ IEEE Photovoltaic Specialists Conference, Philadelphia, USA, June 7-12, 2009, pp. 2256-2259.

Schatten, K. H., Orosz, J. A., 1990. Solar constant secular changes. Solar physics, 125, 179-184, doi:10.1007/BF00154787.

Wald, L., 2007. Solar radiation energy (fundamentals). In Solar Energy Conversion and Photoenergy Systems, edited by Julian Blanco and Sixto Malato, in Encyclopedia of Life Support Systems (EOLSS), Developed under the Auspices of the UNESCO, Eolss Publishers, Oxford ,UK, [http://www.eolss.net]

Walraven, R., 1978. Calculating the position of the Sun. Solar Energy, 20, 393-397. 